\title{
Structure and thermodynamics of square-well and square-shoulder fluids
}

\author{
Andreas Lang $\dagger$, Gerhard Kahl $\uparrow$, Christos N Likos§, Hartmut Löwen§ and \\ Martin Watzlawek§ \\ $\dagger$ Institut für Theoretische Physik, TU Wien, Wiedner Hauptstraße 8-10, A-1040 Wien, Austria \\ \$ CMS, TU Wien, Wiedner Hauptstraße 8-10, A-1040 Wien, Austria \\ $\S$ Institut für Theoretische Physik II, Heinrich-Heine-Universität Düsseldorf, Universitätsstraße 1, \\ D-40225 Düsseldorf, Germany
}

Received 27 July 1999, in final form 2 September 1999

\begin{abstract}
We have reinvestigated the structural and thermodynamic properties of the squarewell and the square-shoulder system using two different theoretical frameworks, i.e., the optimized random-phase approximation and the Rogers-Young integral equation. We discuss the limits of applicability of the respective concepts and compare the results with 'exact' Monte Carlo simulation results and with data obtained from a semi-analytic method proposed by Nezbeda for narrow wells. Using these correlation functions, we study in the framework of the modified-weighteddensity approximation the isostructural solid-solid transition predicted for narrow wells and narrow shoulders.
\end{abstract}

\section{Introduction}

In the 1970s the square-well (SW) system was considered as one of the simplest extensions beyond hard spheres, including both attractive and repulsive forces and representing thus a crude model for a realistic interaction. It therefore was-like the Lennard-Jones liquid — one of the favourite testing grounds of liquid-state theoreticians where it was investigated extensively in computer experiments or within different theoretical frameworks, such as integral equations or perturbation theories (papers stemming from that period are summarized, for instance, in $[1,2]$ ). Improved numerical algorithms (along with better computer performance rates) and more sophisticated theoretical concepts (such as parametrized closure relations for the Ornstein-Zernike-OZ_equations; see [3]) brought realistic pair potentials (such as those of liquid metals) into reach and hence this simple model system quickly lost its attraction. A steadily increasing interest in colloidal fluids has brought this system back into the race, since this model potential is able to mimic the complex interparticle interaction in such systems [4]. Further interest was excited by an isostructural solid-solid transition for the closely related square-shoulder (SS) potential that had been predicted in computer experiments for narrow shoulders $[5,6]$.

Among other papers published on this system during the past few years we point out in particular a HMSA study (i.e. an interpolation between the mean-spherical and the hyperwetted-chain approximation) [7] and a Gibbs ensemble Monte Carlo (GEMC) study [1] that served as reference data for the present study. In this contribution we have complemented the HMSA study by applying the optimized random-phase approximation (ORPA), a successful perturbation theory proposed in the 1970s by Weeks, Chandler and Andersen [8-10] and the 
Rogers-Young (RY) [11] integral equation approach, a parametrized closure relation for the $\mathrm{OZ}$ equations of this system. The ORPA (and its related approximations) is a perturbation theory where the well/shoulder is considered as a perturbation of the hard-sphere reference system; it gives remarkably good results for intermediate and high temperatures, but is bound to fail as the temperature decreases, as the well/shoulder is no longer a small perturbation of the hard core. The RY integral equation, on the other hand, whose closure relation represents a functional interpolation between the Percus-Yevick and the hypernetted-chain approximation is of non-perturbative character and should therefore be applicable in the entire parameter space; nevertheless, we have found in the present study that the solvability of the consistency relation between the virial and the compressibility equation of state (which fixes the interpolation parameter) is also restricted to a fraction of the parameter space.

In the first part of the paper we report on the results for these two frameworks to investigate the structural and thermodynamic properties of SW/SS systems. We discuss the limits of applicability and complement these data with standard Monte Carlo results. Furthermore, we compare our results with those obtained from a semi-analytic Percus-Yevick-based approximation proposed by Nezbeda [12] for narrow wells. We finally discuss in detail the isostructural fcc-fcc transition observed for narrow shoulders and wells $[5,6]$. In contrast to previous theoretical studies [13], we treat the problem in a non-perturbative way and carry out a full mapping of the crystal solid onto an effective liquid in the framework of the modifiedweighted-density approximation (MWDA).

The paper is organized as follows. In the next section we briefly summarize the two theoretical frameworks that are used to investigate the properties of our system, i.e., the ORPA and the RY integral equation. In section 3 we discuss first the results for the square-well system, including structural and thermodynamic data as well as the liquid-gas phase diagram, and then those for the square-shoulder system, focusing there on the isostructural solid-solid transition. The paper is closed with concluding remarks.

\section{Theory}

The methods used in this contribution to investigate the structural and thermodynamic properties of a liquid are based on the Ornstein-Zernike (OZ) equation [3], which relates the direct and the total correlations functions $c(r)$ and $h(r)$ of a system via

$$
h(r)=c(r)+\rho \int \mathrm{d} \boldsymbol{r}^{\prime} c\left(\left|\boldsymbol{r}-\boldsymbol{r}^{\prime}\right|\right) h\left(r^{\prime}\right) .
$$

Here, $\rho$ is the number density of the system; moreover, the relation $g(r)=h(r)+1$ defines the radial distribution function $g(r)$. The static structure factor $S(q)$ is defined through the relation

$$
S(q)=1+\rho \int \mathrm{d} \boldsymbol{r} \exp [-\mathrm{i} \boldsymbol{q} \cdot \boldsymbol{r}] h(r) .
$$

In subsections 2.2 and 2.3 we will present two different approaches that allow the determination of the structure functions $h(r)$ and $g(r)$.

\subsection{The interatomic potential}

The pair potential $\Phi(r)$ of a 'square-well' (SW) or 'square-shoulder' (SS) system is given by

$$
\beta \Phi(r)= \begin{cases}\infty & r<\sigma \\ -\epsilon & \sigma<r<\lambda \sigma \\ 0 & \lambda \sigma<r .\end{cases}
$$


The diameter $\sigma$ defines the (impenetrable) hard-core part of the interaction. In the usual notation, a positive well depth $\epsilon$ represents an attractive SW potential, while a negative $\epsilon$ produces a repulsive SS interaction. The pair potential is furthermore characterized by the well (or shoulder) range $\lambda \sigma$. There are two dimensionless thermodynamic parameters: the 'reduced temperature' $T^{*}=k_{B} T /|\epsilon|$ and the packing fraction of the hard cores, $\eta=(\pi / 6) \rho \sigma^{3}$. We also introduce the dimensionless density $\rho^{*}=\rho \sigma^{3}$.

\subsection{Thermodynamic perturbation theory}

One of the most sophisticated present-day perturbation theories in liquid-state theory is the optimized random-phase approximation (ORPA), which was introduced in the 1970s by Weeks, Chandler and Andersen [8-10,14]. It is based on the following idea: the interatomic potential is split up into a harshly repulsive reference potential and a weak, short-ranged perturbation. The method is particularly appropriate if the reference potential is a hard-core interaction; however, the softness of a potential can be taken into account by the Weeks-Chandler-Andersen (WCA) approach [15] where the soft system is mapped back onto a suitable hard-core system via the blip-function expansion.

To derive the formalism of the ORPA approach it is most convenient to split up, in a similar way to the pair potential $\Phi(r)$, each correlation function into a reference and a perturbation part, labelled by the subscripts ' 0 ' and ' 1 ' respectively, i.e., $c(r)=c_{0}(r)+c_{1}(r)$, etc. The ORPA closure relation for the $\mathrm{OZ}$ equations is then given by

$$
\begin{array}{ll}
c_{1}(r)=-\beta \Phi_{1}(r) & \text { for } r>\sigma \\
h_{1}(r)=0 & \text { for } r<\sigma
\end{array}
$$

where $\sigma$ is the hard-core diameter of the reference potential. The first equation represents the simple random-phase approximation (RPA) which assumes that the long-range behaviour of the direct correlation function is valid for all $r$-values outside the core region. As a consequence of this simple approximation the core condition for the pair distribution function is violated, i.e., $g(r) \neq 0$ for $r \leqslant \sigma$. This deficiency is corrected by the second relation in equation (4).

The OZ equation along with these closure relations can now be treated with standard numerical methods for solving integral equations. However, it can be shown that this integral equation route is equivalent to the solution of a minimization problem, which-in some caseshas turned out to be easier than solving the integral equations themselves. This equivalence has been shown, for instance, by Pastore et al $[16,17]$ : the solution of the integral equation ORPA (in terms of $c(r)$ and $h(r))$ minimizes the following functional:

$$
\mathcal{F}\left[c_{1}\right]=-\frac{1}{(2 \pi)^{3}} \int \mathrm{d} \boldsymbol{q}\left\{\rho S_{0}(q) \tilde{c}_{1}(q)+\ln \left[1-\rho S_{0}(q) \tilde{c}_{1}(q)\right]\right\}
$$

which is a functional of the perturbation part of the direct correlation function $c_{1}(r)$ through its Fourier transform $\tilde{c}_{1}(q) ; S_{0}(q)$ is the static structure factor of the reference system.

In practice, the minimization of the functional $\mathcal{F}$ is done with respect to variations of $c_{1}(r)=-\beta \Phi_{1}(r)$ inside the core, i.e., in a region where finite contributions to the potential do not modify the total pair interaction. In previous implementations of the ORPA $[2,18], \Phi_{1}(r)$ was expanded inside the core in terms of Legendre polynomials and the variational problem was solved with respect to these expansion coefficients. Thanks to modern tools and to the performance of modern computers it has now become possible to discretize $\Phi_{1}(r)$ inside the core region on a grid of typically 100 points and to minimize the functional $\mathcal{F}\left[c_{1}\right]$ with respect to these discrete function values. The appropriate numerical tool for this minimization problem is the steepest-descent algorithm in view of the fact that the gradient of the functional $\mathcal{F}$ with 
respect to $c_{1}(r)$ is given by the total correlation function, i.e.,

$$
\frac{\delta \mathcal{F}\left[c_{1}\right]}{\delta c_{1}}(r)=\rho h_{1}(r)
$$

where $\delta$ denotes a functional derivative (for details see again [16]).

In our case the reference system is a hard-sphere (HS) system. The relevant information about its structural and thermodynamic properties can be obtained via two routes: either from the analytic solution of the Percus-Yevick (PY) approximation for HS [19] or from the VerletWeis (VW) parametrization [20] which reproduce results from computer experiments very accurately. The crucial difference between these two routes is that the PY $c_{0}(r)$ is zero outside the core, while the VW $c_{0}(r)$ does not vanish in this region. Hence, the ORPA in combination with the PY parametrization for the reference system becomes equivalent to the mean-spherical approximation (MSA) [3] and we will carry on using this notation, while 'ORPA' now means that we use a VW parametrization for the properties of the HS reference system. This difference will in particular be important when we discuss thermodynamic properties.

Once this minimization problem is solved and the full set of correlation functions is known one can proceed to more sophisticated extensions of the ORPA: a closer analysis of the graphtheoretical representation of the correlation functions shows [9] that inclusion of the next-order graphs yields the following expression for the pair distribution function:

$$
g(r)=g_{0}(r) \exp \left[h_{1}(r)\right]
$$

to which we refer to as the optimized-cluster theory (OCT). In a semi-heuristic way, a modified version of (7) has been introduced, the linearized exponential approximation (LEXP) [21], where

$$
g(r)=g_{0}(r)\left[1+h_{1}(r)\right] .
$$

Given the structure of the system in terms of the correlation functions, the thermodynamic properties can be calculated. The excess internal energy $U^{\mathrm{ex}}$ and the 'fluctuation' isothermal compressibility $\chi^{\text {fluc }}$ are obtained via standard relations, i.e.,

$$
U^{*}=\frac{\beta U^{\mathrm{ex}}}{N}=-2 \pi \epsilon \rho \int_{\sigma}^{\lambda \sigma} \mathrm{d} r r^{2} g(r)
$$

and

$$
\rho k_{\mathrm{B}} T \chi^{\text {fluc }}=S(0) \text {. }
$$

The calculation of the virial pressure $P_{\mathrm{vir}}$ is a more delicate task: starting from the general expression

$$
\frac{\beta P_{\text {vir }}}{\rho}=1-\frac{2}{3} \pi \beta \rho \int_{0}^{\infty} \mathrm{d} r r^{3} \Phi^{\prime}(r) g(r)
$$

particular care has to be taken for discontinuities both in $g(r)$ and in the derivative of the interaction, i.e., $\Phi^{\prime}(r)$ for $r=\sigma$ and $r=\lambda \sigma$. As described in detail in reference [22], one finds the following expression, which is exact and whose derivation rests on the continuity of the function $y(r)=g(r) \exp [\beta \Phi(r)]$ :

$$
P_{\text {vir }}^{*}=1+\frac{2 \pi}{3} \rho \sigma^{3}\left\{g\left(\sigma^{+}\right)+\lambda^{3}\left[g\left(\lambda \sigma^{+}\right)-g\left(\lambda \sigma^{-}\right)\right]\right\} .
$$

While the continuity of $y(r)$ is preserved in the OCT (as well as in the integral equation theories to be discussed in the following subsection), this property is violated in the ORPA, the MSA and the LEXP. For these theories, the pressure is given instead by the approximate relation [22]:

$$
P_{\text {vir }}^{*}=1+\frac{2 \pi}{3} \rho \sigma^{3}\left\{g\left(\sigma^{+}\right)-\frac{1}{2} \lambda^{3} \beta \epsilon\left[g\left(\lambda \sigma^{+}\right)+g\left(\lambda \sigma^{-}\right)\right]\right\} .
$$


One of the attractive features of the ORPA (and related approximations) is the fact that closed expressions can be given for the free energy $A$, which avoids tedious thermodynamic integrations. One finds for the ORPA and the MSA

$$
A^{*}=\frac{\beta}{N} A=A_{0}^{*}+A_{\mathrm{HTA}}^{*}+A_{\mathrm{ORPA}}^{*}
$$

where $A_{0}^{*}$ is the reduced (dimensionless) free energy of the HS reference system (PY or VW parametrization), $A_{\mathrm{HTA}}^{*}$ is the high-temperature contribution, given by

$$
A_{\mathrm{HTA}}^{*}=2 \pi \rho \int_{0}^{\infty} \mathrm{d} r r^{2} \beta \Phi_{1}(r) g_{0}(r)
$$

and $A_{\text {ORPA }}^{*}$ is found to be

$$
A_{\mathrm{ORPA}}^{*}=-\frac{1}{2 \rho} \mathcal{F}\left[c_{1}\right] .
$$

Inclusion of higher-order terms within the OCT framework leads to the following expression for $A^{*}$ :

$$
A_{\mathrm{OCT}}^{*}=A_{0}^{*}+A_{\mathrm{HTA}}^{*}+A_{\mathrm{ORPA}}^{*}+B_{2}^{*}
$$

with

$$
B_{2}^{*}=-2 \pi \rho \int_{\sigma}^{\infty} \mathrm{d} r r^{2}\left\{g_{0}(r)\left[\exp \left[h_{1}(r)\right]-h_{1}(r)-1\right]-\frac{1}{2} h_{1}^{2}(r)\right\} .
$$

Finally, the chemical potential $\mu$ can be given as a closed relation in the MSA; for the other approximations we have to use numerical differentiations (the Maxwell relation) or we exploit the Gibbs-Duhem relation, i.e.,

$$
\mu^{*}=A^{*}+P^{*} \text {. }
$$

In principle, different routes to a thermodynamic quantity should give the same results. However, due to the approximations assumed in the derivation of a closure relation we are confronted with the fact that the results now do depend on the route by which they have been calculated. This feature, known in the literature as thermodynamic inconsistency, will be dealt with in section 3.1.2.

\subsection{Integral equations}

An alternative approach to the calculation of the structural and thermodynamic properties of classical liquids is offered by employing integral equation theories (IETs). Unlike the ORPA, such theories are non-perturbative in character, i.e., they do not rely on any separation of the pair potential into a reference and a perturbation, but rather they treat the whole pair interaction on an equal footing. An obvious advantage of IETs compared with the ORPA is that, in principle, they remain valid at all temperatures whereas the ORPA closure (equation (4)) yields invariably a diverging direct correlation function for $r>\sigma$ at $T=0$.

The starting point of any approximate integral equation theory is the exact relation connecting the radial distribution function $g(r)$ to the direct correlation function $c(r)$ and involving the bridge function $B(r)$ :

$$
g(r)=\exp \{-\beta \Phi(r)+g(r)-1-c(r)-B(r)\}
$$

where $B(r)$ stands for the sum of all elementary diagrams that are not nodal [3]. As $B(r)$ is not known, the various approximate IETs can be regarded as approximations of this quantity. In this way, an additional closure involving only $g(r)$ and $c(r)$ is supplemented to the OrnsteinZernike relation (equation (1)) and the system becomes, in principle, solvable. 
The simplest and most frequently used IETs are the hypernetted-chain (HNC) and the Percus-Yevick (PY) closure. In the HNC one simply sets $B(r)=0$, obtaining the closure

$$
g(r)=\exp \{-\beta \Phi(r)+g(r)-1-c(r)\}
$$

On the other hand, the PY closure can be regarded as a linearization of the HNC scheme regarding the term $g(r)-1-c(r)$ in the exponential and reads as

$$
g(r)=\exp \{-\beta \Phi(r)\}[g(r)-c(r)]
$$

corresponding to the following approximation for the bridge function:

$$
B_{\mathrm{PY}}(r)=[g(r)-c(r)]-1-\ln [g(r)-c(r)] .
$$

Both the HNC and the PY closures, being approximate in character, lead to the problem of thermodynamic inconsistency mentioned above. In order to overcome this difficulty, more sophisticated schemes have been proposed that have the freedom of one or more adjustable parameters which are then chosen in such a way that thermodynamic consistency is achieved. Among the most popular approaches are the modified HNC (MHNC) of Rosenfeld and Ashcroft [23], the HMSA of Bergenholtz et al [7] and the Rogers-Young (RY) closure [11]. In the latter, one replaces the exact relation (20) above with the closure

$$
g(r)=\exp \{-\beta \Phi(r)\}\left[1+\frac{\exp \{\gamma(r) f(r)\}-1}{f(r)}\right]
$$

where $\gamma(r)=g(r)-c(r)-1$ and $f(r)$ is a 'mixing function' involving a parameter $\alpha$ and taken to have the form

$$
f(r)=1-\exp (-\alpha r) .
$$

It is straightforward to verify that for $\alpha=0$ the RY closure reduces to PY and for $\alpha \rightarrow \infty$ it reduces to the HNC. The parameter $\alpha$ is chosen in such a way that thermodynamic consistency between the 'virial' and the 'compressibility' routes to the pressure of the system is achieved.

The Rogers-Young closure has been proven to be very accurate for a number of model systems with variable pair interactions, ranging from hard spheres and inverse-power potentials [11] to ultrasoft logarithmic interactions [24]. Here we want to check the validity of the RY closure for square-well and square-shoulder potentials and check, in particular, how the existence of an attractive part in the potential affects the quality of the RY results. In table 1 (below) and table 3 (see later) we present a summary of the thermodynamic parameters for which we carried out the RY closure.

Table 1. The combination of thermodynamic parameters for which the Rogers-Young and the ORPA closures were solved, for the square-well fluid.

\begin{tabular}{lll}
\hline$\lambda$ & $T^{*}$ & $\rho^{*}$ \\
\hline 1.50 & 1.0 & 0.80 \\
1.03 & 1.0 & 0.80 \\
1.03 & 0.5 & 0.80 \\
\hline
\end{tabular}

\subsection{The Nezbeda solution}

It should also be noted that Nezbeda [12] has derived an approximate analytic solution for the direct correlation function within the PY approximation. The Nezbeda approximation is valid for short-range wells or shoulders, typically $\lambda \leqslant 1.05$ only; its advantage is that all the 
parameters that enter the expressions for the correlation functions can be given analytically as functions of density and temperature; for a summary see reference [25]. Hence, for short-range potentials we are also going to present results from the Nezbeda approximation in order to provide a comparison.

\subsection{Simulations}

Finally, in order to assess the quality of all of the approximations mentioned above, we have also carried out standard Monte Carlo simulations [26] in the constant- $N V T$ ensemble. All runs were performed in a cubic box containing 500 particles and using periodic boundary conditions. We calculate the radial distribution function $g(r)$ and the structure factor $S(q)$ 'on the fly'.

\section{Results}

\section{1. 'Square-well' systems}

3.1.1. Structure. Here, we will only present results for the pair distribution function $g(r)$. The combination of thermodynamic parameters for which the ORPA and Rogers-Young closures were solved are shown in table 1 . We begin with a square-well fluid having a width parameter $\lambda=1.5$. In figure 1 we show results for $g(r)$ at reduced temperature $T^{*}=1$. As can be seen in figure 1(a), the ORPA yields results which are in good agreement with simulation and actually superior to the OCT approximation.

The situation with the RY closure is completely different: at density $\rho^{*}=0.8$, there is no thermodynamically self-consistent solution to this closure. This failure was also observed earlier in a different, albeit similar closure, for the same system, by Bergenholtz et al [7]. The reason for the lack of existence of a solution is the following: the RY closure is a mixture of the Percus-Yevick (PY) and the hypernetted-chain (HNC) closures. In a case where it works (e.g., hard spheres) the difference between the 'fluctuation' and the 'virial' compressibilities, $\chi^{\text {fluc }}=\beta S(0) / \rho$ and $\chi^{\text {vir }}=[\rho \partial P / \partial \rho]^{-1}$, has two different signs in these two different closures. In particular, the PY closure typically predicts too low pressures, with the result that the virial compressibility is too high and the quantity $\chi^{\text {fluc }}-\chi^{\text {vir }}$ is negative. On the other hand, the HNC predicts too high pressures and the quantity $\chi^{\text {fluc }}-\chi^{\text {vir }}$ is positive. With reference to figures 1(a) and 1(b), we now see that the major 'jump' in $g(r)$ (at $r=\sigma$ ), whose magnitude gives the dominant contribution to the virial pressure, turns out to be higher than the simulation result in both the PY and the HNC closures. Hence, both yield a positive value for the difference $\chi^{\text {fluc }}-\chi^{\text {vir }}$ and a solution of the RY closure does not exist. This is the same mechanism that brings about the failure of the HMSA closure of Bergenholtz et al [7].

The self-consistency parameter $\alpha$ of the RY closure cannot attain negative values, as is clear from equation (25). We can, therefore, trace out the domain in thermodynamic space where the RY closure fails, by keeping track of the value of $\alpha$ as a function of density and temperature and working out the locus of points where $\alpha=0$. In figure 2 we show the parameter $\alpha$ as a function of inverse temperature for the $\lambda=1.5$ square-well fluid for a number of reduced densities. It can be seen that with decreasing temperature the mixing parameter decreases for all values of the reduced density. Beyond the point where $\alpha=0$, a solution of the RY closure is no longer feasible. In figure 3 the locus of points $\alpha=0$ in conjunction with the fluid part of the phase diagram of the system, as calculated within the ORPA, is shown. The region below the broken line is the region where the RY closure has no solution.

For narrow-square-well systems, $\lambda=1.03$, the solution of the RY closure is 

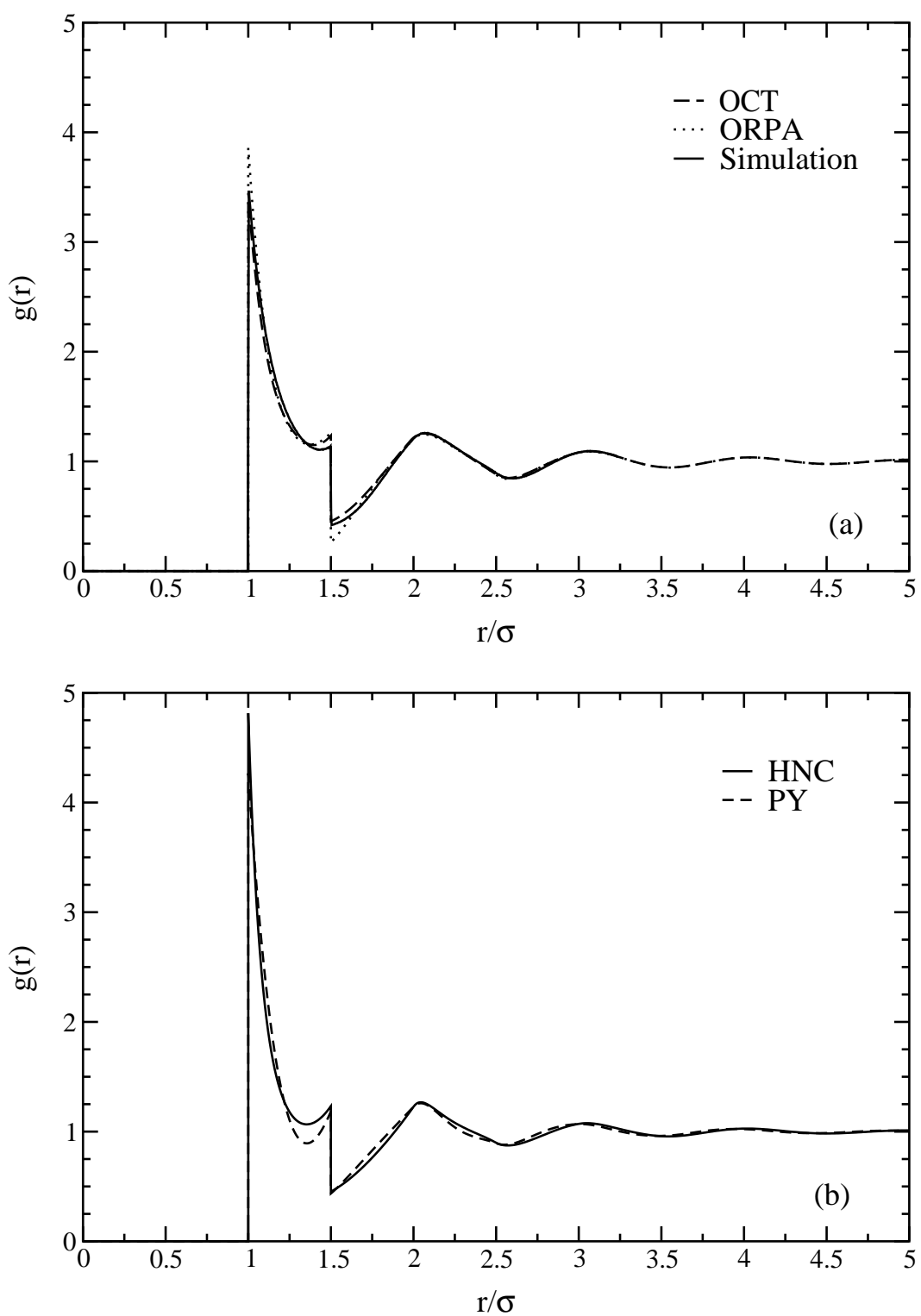

Figure 1. (a) The pair distribution function for $\lambda=1.5, \rho^{*}=0.8, T^{*}=1$ for the square-well potential. ORPA/OCT and simulation results. (b) The same as (a) but for Percus-Yevick and hypernetted-chain results.

unproblematic. In figure 4 we show results for $g(r)$ at two different temperatures, $T^{*}=1.0$ and 0.5 , where it can be seen that the Nezbeda and RY solutions run very close to each other. The simulation result is practically indistinguishable for the RY result and is thus not shown, in order not to overcrowd the figure. The ORPA and OCT approximations, however, predict too low and too high values for $g(r)$ in the narrow well, respectively. We conclude that the RY closure develops problems when the range of the attractive potential grows, but for narrow wells it still yields results which are the most reliable ones. 


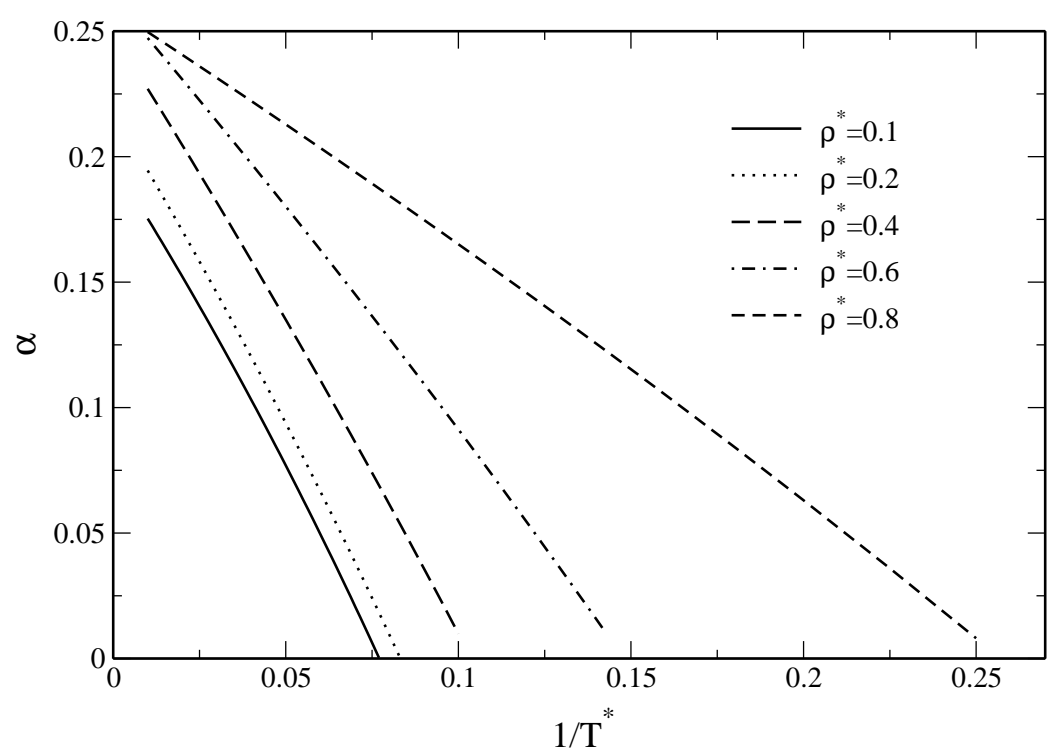

Figure 2. The mixing parameter $\alpha$ (see equation (25)) of the Rogers-Young closure for the $\lambda=1.5$ square-well fluid as a function of inverse reduced temperature and for a number of different densities.

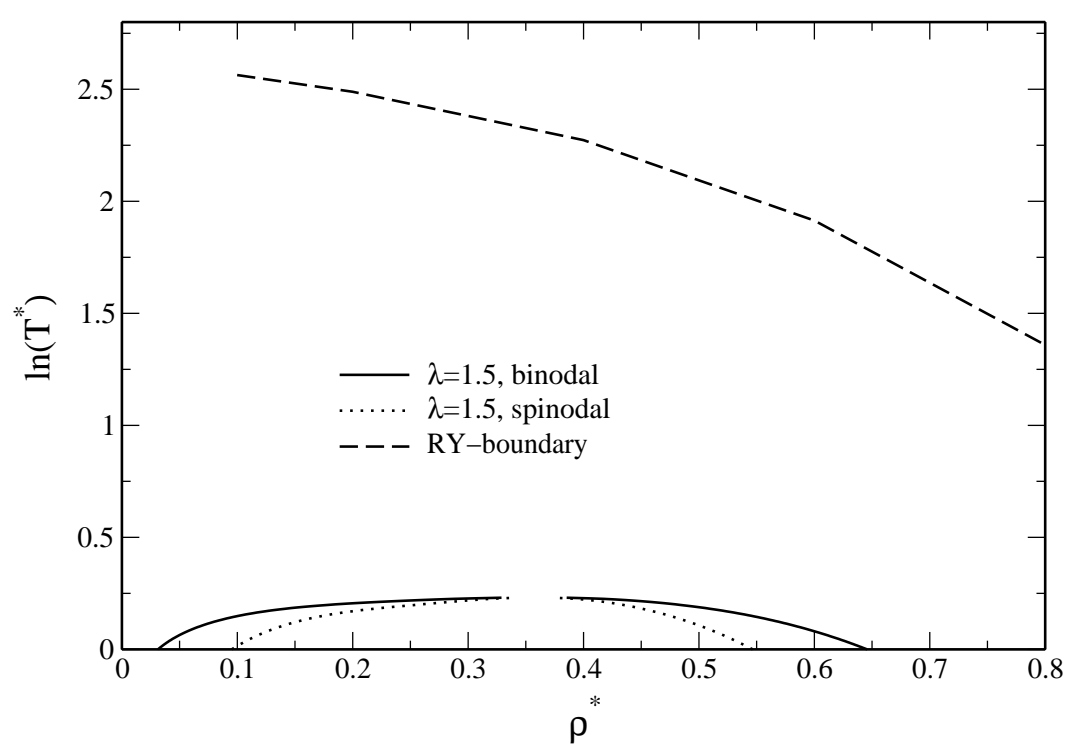

Figure 3. The fluid part of the phase diagram of the $\lambda=1.5$ square-well fluid as calculated within the ORPA and the locus of points where the RY closure has $\alpha=0$ (RY boundary). Above this boundary $\alpha>0$ and the RY closure has a solution, but below a solution is not possible.

3.1.2. Thermodynamic properties. We have examined the thermodynamic properties for the square-well potential for a number of parameters, shown, together with the results, in table 2. The calculation was carried out using the ORPA and OCT approximations. In figure 5(a) we plot the results for the pressure, obtained via the virial and the energy route within the 

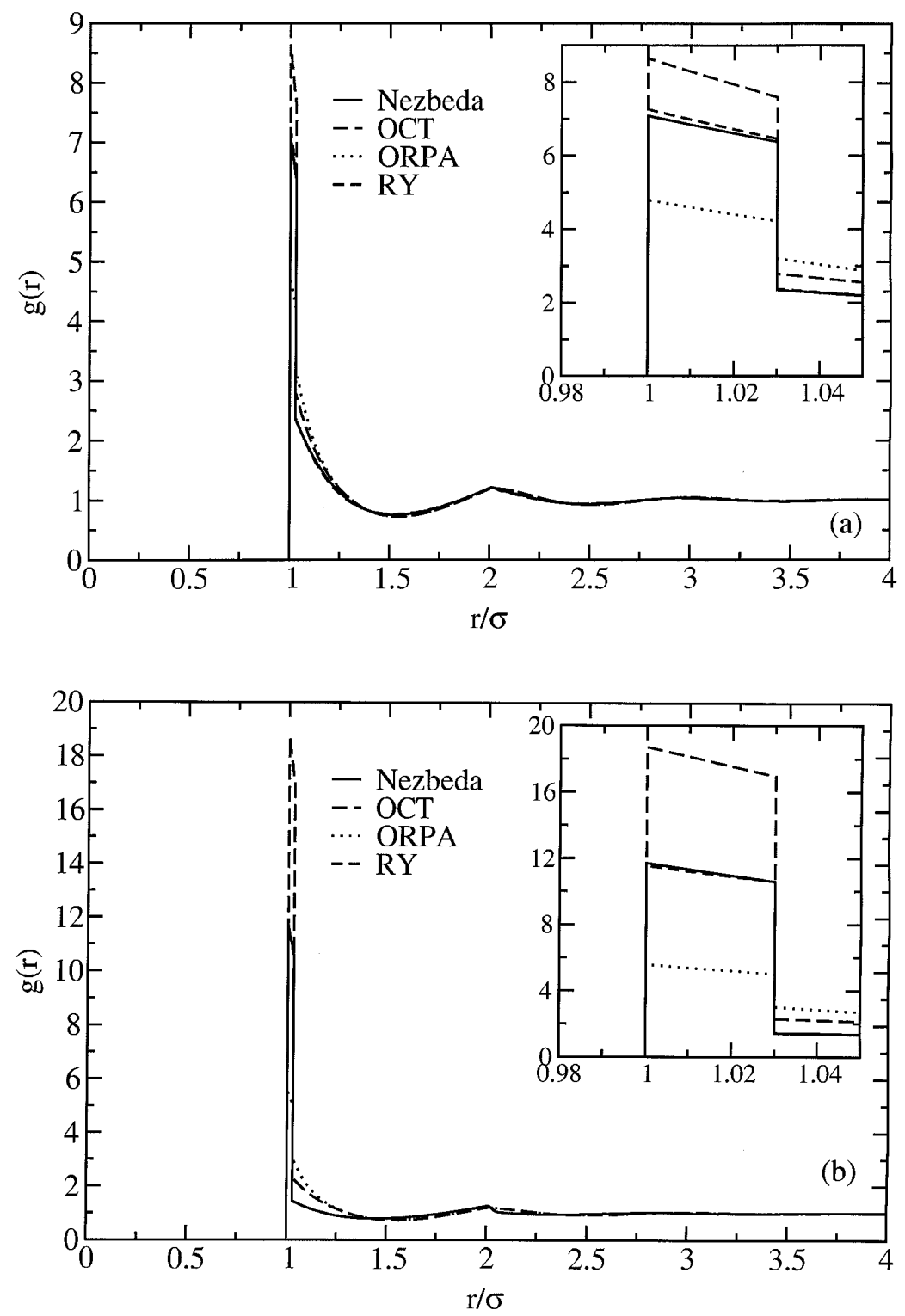

Figure 4. The pair distribution function for the $\lambda=1.03$ square-well system at reduced density $\rho^{*}=0.8$. Comparison between the ORPA/OCT, Rogers-Young and Nezbeda solutions; (a) $T^{*}=1.0$; (b) $T^{*}=0.5$

OCT for a SW system of range $\lambda=1.5$ and three different temperatures $T^{*}$; the error bars indicate the difference between the two pressure values and hence the degree of thermodynamic inconsistency. Figure 5(b) presents similar results for the compressibility $\chi_{T}$, obtained via the fluctuation and the compressibility route for a SS system with a range of $\lambda=1.2$. We observe that the thermodynamic self-consistency gets worse as the temperature decreases. In table 2 we present results for three special choices of systems parameters: remarkable is the total failure (with an error of $\approx 200 \%$ ) for the pressure self-consistency of the $\lambda=1.03$ and 
Table 2. Thermodynamic properties for various square-well liquids at reduced density $\rho^{*}=0.8$. $P_{\text {vir }}^{*}$ and $P_{\mathrm{e}}^{*}$ denote the pressure calculated by the virial (equation (12)) and energy routes; $U^{*}$ and $U_{\mathrm{e}}^{*}$ are the excess internal energy per particle calculated by the virial (equation (9)) and energy routes; $\chi^{\text {fluc }}$ and $\chi^{\text {vir }}$ denote the 'fluctuation' and 'virial' isothermal compressibilities, respectively. (a) ORPA results; (b) OCT results.

\begin{tabular}{|c|c|c|c|c|c|c|c|}
\hline$\lambda$ & $T^{*}$ & $P_{\mathrm{vir}}^{*}$ & $P_{\mathrm{e}}^{*}$ & $U^{*}$ & $U_{\mathrm{e}}^{*}$ & $\chi^{\text {fluc }}$ & $\chi^{\text {vir }}$ \\
\hline \multicolumn{8}{|l|}{ (a) } \\
\hline 1.50 & 1.0 & 3.158 & 2.581 & -3.1179 & -3.1140 & 0.0715 & 0.0505 \\
\hline 1.03 & 1.0 & 2.225 & 6.158 & 0.5343 & 0.5371 & 0.0504 & 0.1827 \\
\hline 1.03 & 0.5 & $-4.397^{\dagger}$ & 4.498 & -0.0945 & -0.0833 & 0.0557 & -0.0846 \\
\hline \multicolumn{8}{|l|}{ (b) } \\
\hline 1.50 & 1.0 & 2.267 & 2.868 & -2.9802 & -3.0696 & 0.0715 & 0.0505 \\
\hline 1.03 & 1.0 & 6.705 & 5.693 & 0.1601 & 0.2121 & 0.0504 & 0.0524 \\
\hline 1.03 & 0.5 & 5.513 & 2.208 & -2.6909 & -2.3028 & 0.0557 & 0.0589 \\
\hline
\end{tabular}

\footnotetext{
$\dagger$ For the virial pressure of the ORPA we use equation (13) instead of (12).
}

$T^{*}=0.5$ system. Using the double-tangent construction we have finally calculated the liquidgas phase diagram of SW systems for different values of well range $\lambda$. We have compared our results with the GEMC data of [1] and observe that the critical data differ by up to 5\%, which is not surprising since it is well known [27] that conventional liquid-state theories are not appropriate for giving quantitative predictions of the critical data. Below the critical point the phase separation curves are in good agreement with simulation data.

\section{2. 'Square-shoulder' systems}

3.2.1. Structure. The combinations of thermodynamic parameters for which we solved the ORPA and RY closures for the square-shoulder systems are summarized in table 3. Results for $g(r)$ for two different values of $\lambda(\lambda=1.5$ and 1.2) and at two different temperatures are shown in figures 6 and 7. It can now be seen that the RY closure delivers results which are in perfect agreement with simulation. We have further explored the thermodynamic

Table 3. The combination of thermodynamic parameters for which the Rogers-Young closure was solved, for the square-shoulder fluid.

\begin{tabular}{lll}
\hline$\lambda$ & $T^{*}$ & $\eta$ \\
\hline 1.50 & 1.0 & 0.10 \\
& & 0.25 \\
& & 0.40 \\
& 0.5 & 0.10 \\
& & 0.25 \\
& & 0.40 \\
\hline 1.20 & 1.0 & 0.10 \\
& & 0.25 \\
& & 0.40 \\
& & \\
& 0.5 & 0.10 \\
& & 0.25 \\
& & 0.40 \\
\hline
\end{tabular}



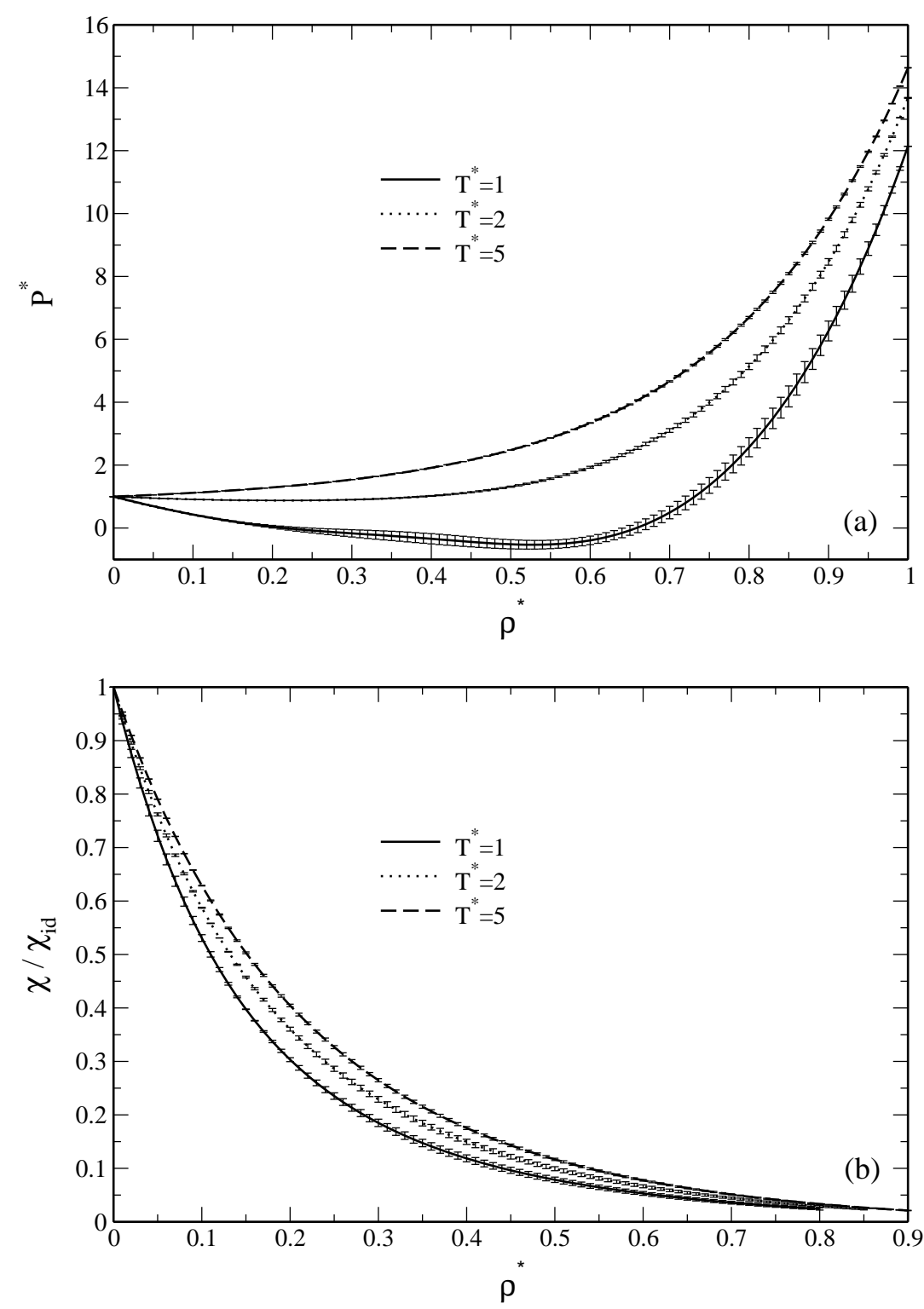

Figure 5. (a) The reduced dimensionless pressure $P^{*}$ calculated for a SW system of range $\lambda=1.5$ for three different temperatures $T^{*}$ as indicated, calculated via the virial and the energy route. The lines indicate the average of the values for the two routes and the error bars the difference between these two values. (b) The reduced isothermal compressibility $\chi / \chi_{\text {id }}\left(\chi_{\text {id }}=\beta / \rho\right)$ calculated for a SS system of range $\lambda=1.2$ for three different temperatures $T^{*}$ as indicated, calculated via the compressibility and the virial route. The lines denote the average of the values for the two routes and the error bars the difference between these two values.

space and we were always able to find a self-consistent solution to the RY closure. We conclude, therefore, that for purely repulsive potentials the RY closure is problem free and, in addition, yields results of excellent quality, when a comparison with the simulation is made. 

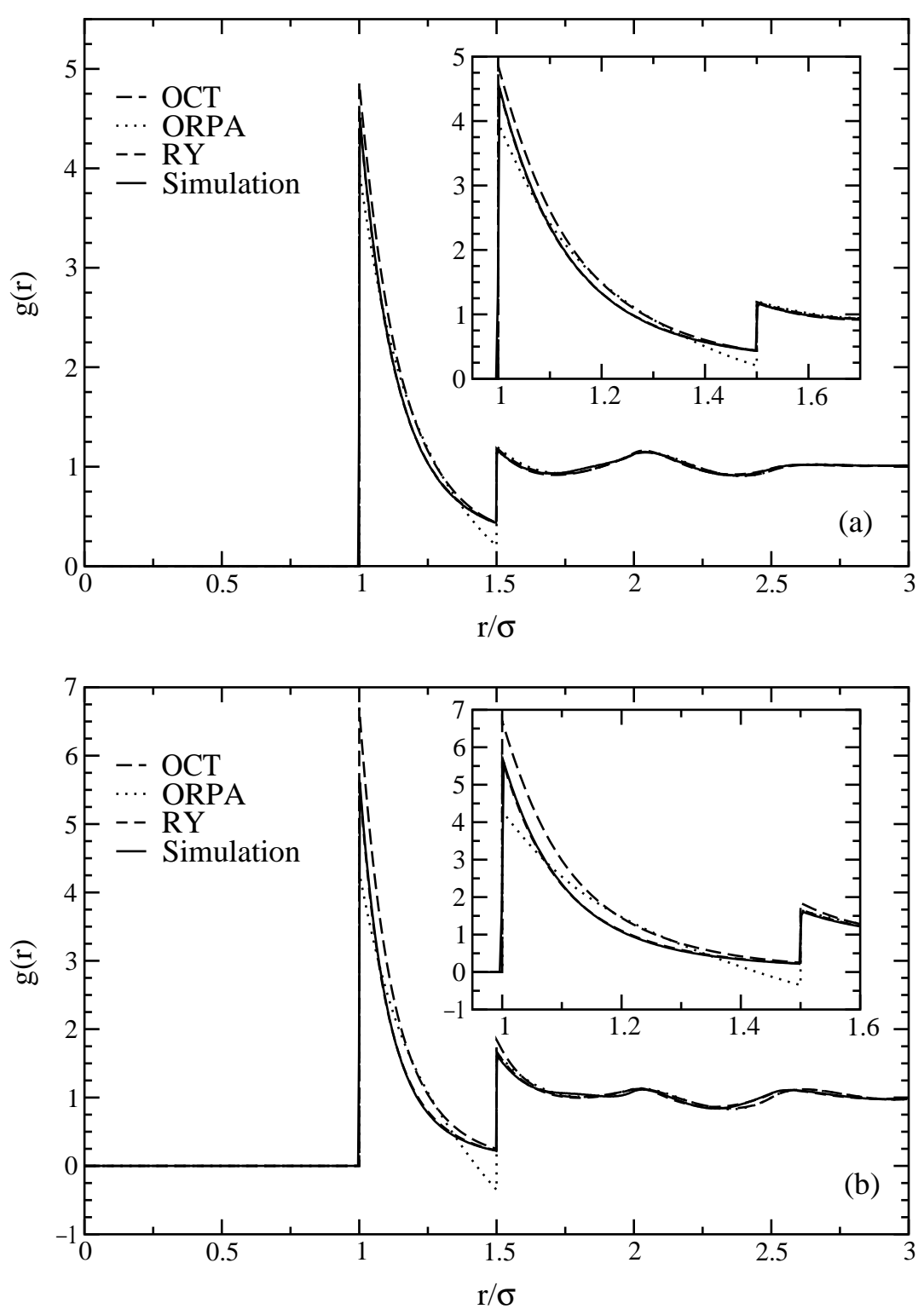

Figure 6. (a) The pair distribution function for the $\lambda=1.5$ square-shoulder fluid at packing fraction $\eta=0.4$. Comparison between the ORPA/OCT, Rogers-Young and simulation approaches; (a) $T^{*}=1.0$; (b) $T^{*}=0.5$. Note that the RY results are practically indistinguishable from the Monte Carlo data.

The ORPA and OCT approximations, on the other hand, do not yield satisfactory agreement with simulation. In one case, $\lambda=1.5, T^{*}=0.5$ and $\eta=0.4$, the ORPA even predicts a region where $g(r)$ is negative (see figure 6(b)), a clear physical impossibility. The reason for this failure can be traced back to the perturbative nature of the ORPA. Indeed, from the defining equations for the ORPA (see equation (4)) it is clear that the latter is a high-temperature approximation which is bound to fail at sufficiently low temperatures as the 

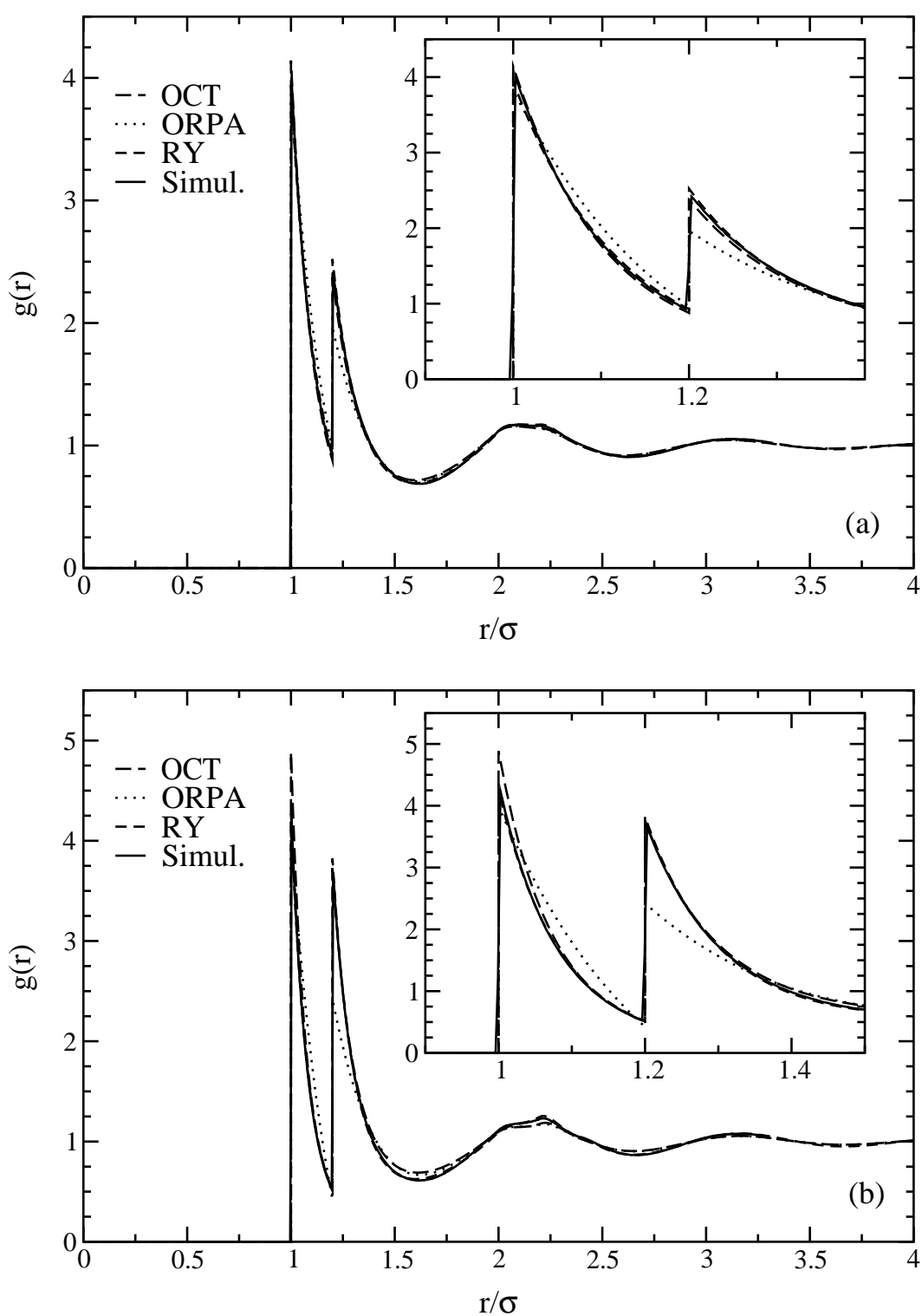

Figure 7. As figure 6, but for $\lambda=1.2$.

quantity $-\beta \Phi_{1}(r)$ will tend to minus infinity for the case of the square-shoulder potential. In figures 8 and 9 we delineate the region in the density-temperature plane where the ORPA develops negative parts for the function $g(r)$ in the case of square-shoulder systems with $\lambda=1.5$ and 1.2 , respectively.

3.2.2. Thermodynamic properties. In the square-shoulder system, the absence of attractive parts in the interaction potential means that there exists only one fluid phase, i.e., there is no liquid-gas separation. However, for narrow shoulders there appears in the solid region 


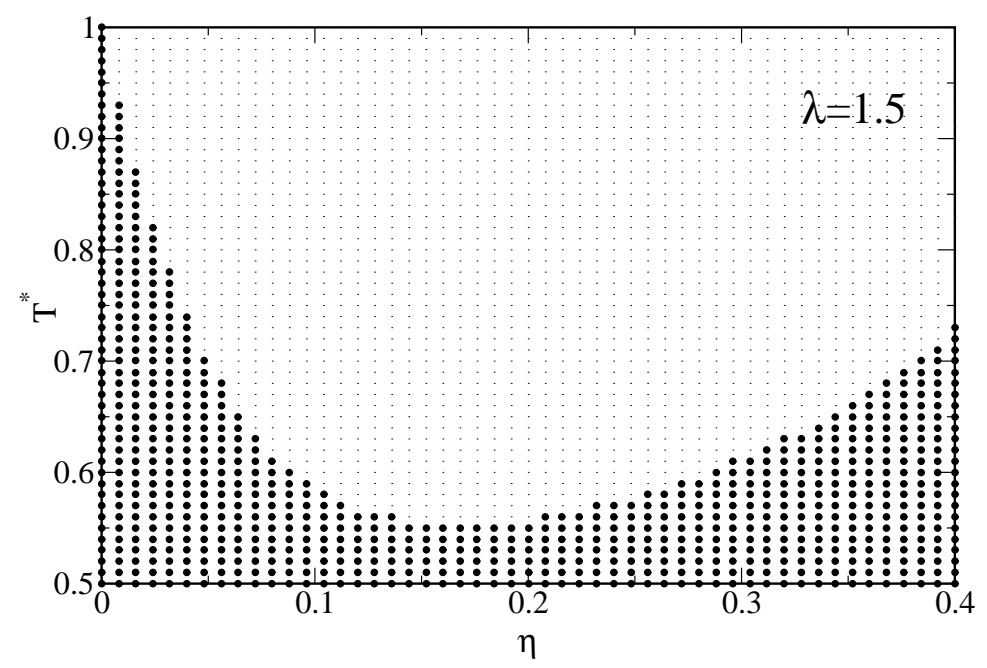

Figure 8. The region of failure of the ORPA (see the text), for the square-shoulder potential, with $\lambda=1.5$. The region is denoted by the dots.

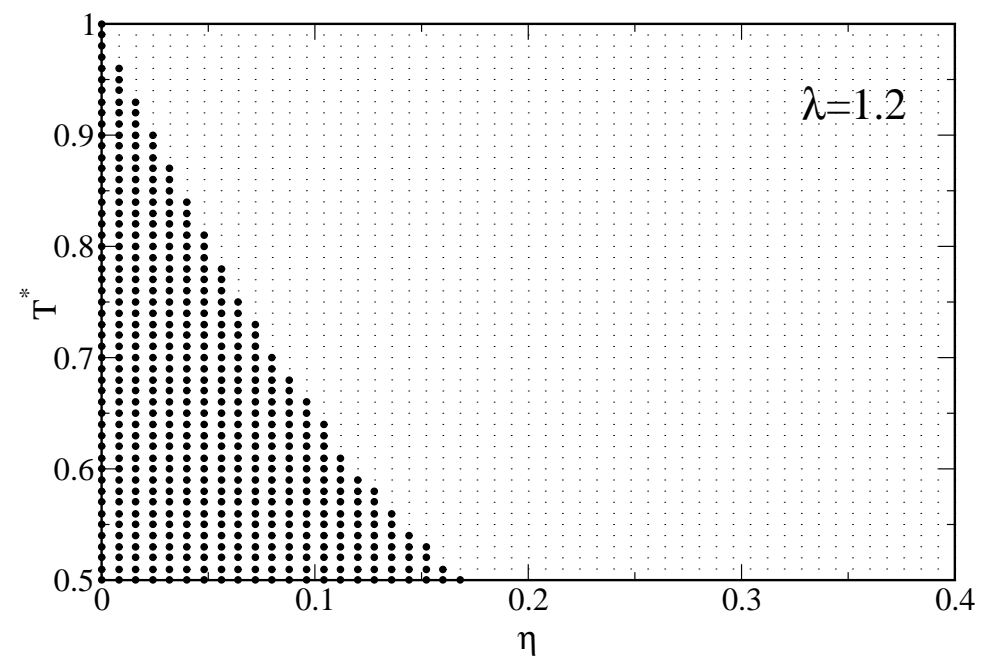

Figure 9. As figure 8, but for $\lambda=1.2$.

of the phase diagram a new type of phase coexistence, namely an isostructural fcc-fcc phase transition, terminating in a critical point. This transition was discovered in simulational work by Bolhuis and Frenkel and it exists both for narrow-square-shoulder systems [5] and for narrowsquare-well systems [6]. This isostructural transition was studied recently in the framework of density functional theory by Denton and Löwen [13]. Thereby, a perturbative approach was employed, where the interaction potential was separated into a reference, hard-sphere part and a perturbation. The free energy of the inhomogeneous (crystalline) system was calculated by employing the modified-weighted-density approximation (MWDA) [28] for the reference part and a mean-field-type approach for the perturbation. The results obtained using this approach 
were in good agreement with simulation [13].

Here, we would like to treat the problem in a non-perturbative fashion, i.e., without separation of the interaction potential into a reference and a perturbation part, and carry out a full mapping of the crystalline solid onto an effective liquid in the framework of the MWDA. This approach has already been carried out for the case of square-well potentials [25]. The system at hand presents no possible complications with the mapping, as there exists only one stable fluid phase.

The MWDA amounts to an approximation of the excess free energy $\beta F_{\mathrm{ex}}$ of the solid having packing fraction $\eta_{s}$ through the excess free energy of an effective liquid having a packing fraction $\hat{\eta}$, i.e.:

$$
\frac{\beta F_{\mathrm{ex}}[\rho] \sigma^{3}}{V}=\eta_{s} \frac{f(\hat{\eta})}{\hat{\eta}}
$$

where $f(\eta)$ is $\sigma^{3}$ times the excess free energy per unit volume of the uniform fluid. With $\rho(\boldsymbol{r})$ being the spatially modulated one-particle density of the crystal, the self-consistency condition that determines the weighted packing fraction $\hat{\eta}$ in terms of $\rho(\boldsymbol{r})$ reads in real space as [25]

$$
\begin{aligned}
\hat{\eta}=\eta_{s}\left[1+\frac{18 \hat{\eta}^{2} \tilde{c}(k=0 ; \hat{\eta})}{\pi^{2}\left(\hat{\eta} f^{\prime}(\hat{\eta})-f(\hat{\eta})\right)}\right] & \\
& \quad-\frac{6 \hat{\eta}^{2}}{\pi\left(\hat{\eta} f^{\prime}(\hat{\eta})-f(\hat{\eta})\right)}\left(\frac{1}{2 N} \iint \rho(\boldsymbol{r}) \rho\left(\boldsymbol{r}^{\prime}\right) c\left(\left|\boldsymbol{r}-\boldsymbol{r}^{\prime}\right| ; \hat{\eta}\right) \mathrm{d} \boldsymbol{r} \mathrm{d} \boldsymbol{r}^{\prime}\right) .
\end{aligned}
$$

In equation (27) above, $\tilde{c}(k ; \eta)$ is $\sigma^{3}$ times the Fourier transform of the direct correlation function of a liquid at packing fraction $\eta$, the prime on $f$ denotes a derivative with respect to $\eta$ and $N$ is the number of particles in the system.

We have performed the MWDA iteration using, at first, the Nezbeda solution for $c(r ; \eta)$ as input for the liquid. The result is a serious overestimation of the critical temperature for the isostructural transition. We obtain, for $1.04 \leqslant \lambda \leqslant 1.08, T_{c}^{*} \approx 6$, in disagreement with the result from simulations [5], $T_{c}^{*} \approx 1.5$. On the other hand, if we use the ORPA result for $c(r ; \eta)$ as input, then the critical temperature turns out to be between 0.9 and 1.3 , in much better agreement with simulation. The ORPA phase diagram is displayed in figure 10 .

This extreme sensitivity of $T_{c}^{*}$ to the liquid-state input requires some explanation. The basic idea behind the MWDA is that the effective liquid whose excess free energy equals that of the solid has a packing fraction $\hat{\eta}$ which is much lower that $\eta_{s}$. Indeed, the solid, being highly inhomogeneous, pays a high price in ideal free energy, which disfavours spatial modulations, and a relatively low price in excess free energy. In other words, the MWDA is self-consistent, if the value of the effective packing fraction $\hat{\eta}$ that corresponds to a strongly modulated solid is low. Referring to equation (27) above, we observe the following: the contribution of the second term on the rhs consists of sums over shells in real space, the zeroth shell being included. The contributions from the non-zero shells (i.e., first, second etc neighbours of a given site) are practically vanishing if the Nezbeda solution for $c(r ; \eta)$ is used, as in the approximation $c(r ; \eta)$ is identically zero for $r>\lambda \sigma$. In reality, however, the function $c(r ; \eta)$ has a 'tail' in the region $r>\lambda \sigma$, where it attains positive values. This tail is reproduced in both the ORPA and the RY solution; see figure 11. As the tail is positive, $\rho(r)$ is also positive and so is the coefficient of the double integral on the rhs of equation (27); it turns out that if the Nezbeda solution is used, certain negative contributions to the determination of $\hat{\eta}$ are left out. This yields an effective packing fraction $\hat{\eta}$ which is too high. Indeed, for $\lambda=1.05$ and $T^{*}=1.0$, we find, typically, $0.40<\hat{\eta}<0.55$ in the region of fcc-fcc coexistence. For such high values of $\eta$, the validity of the PY approximation (inherent in the Nezbeda solution) is questionable. Moreover, the 


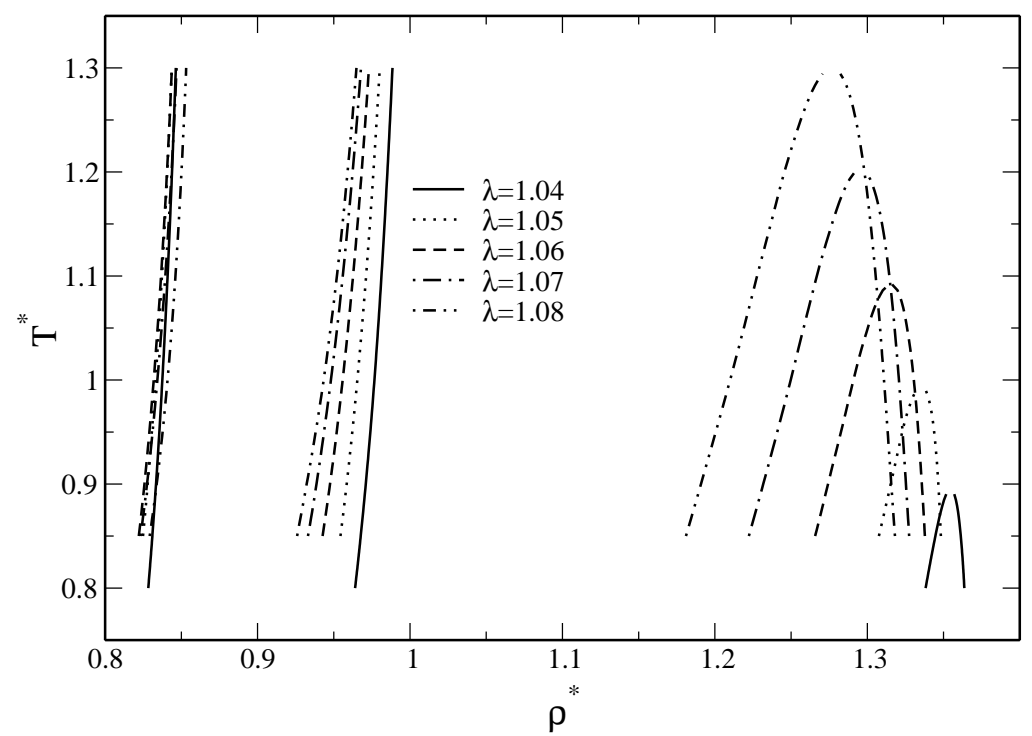

Figure 10. The phase diagram of square-shoulder liquids for various different values of $\lambda$. The phase boundaries were obtained using the MWDA and the ORPA solution for the liquid state of the system.

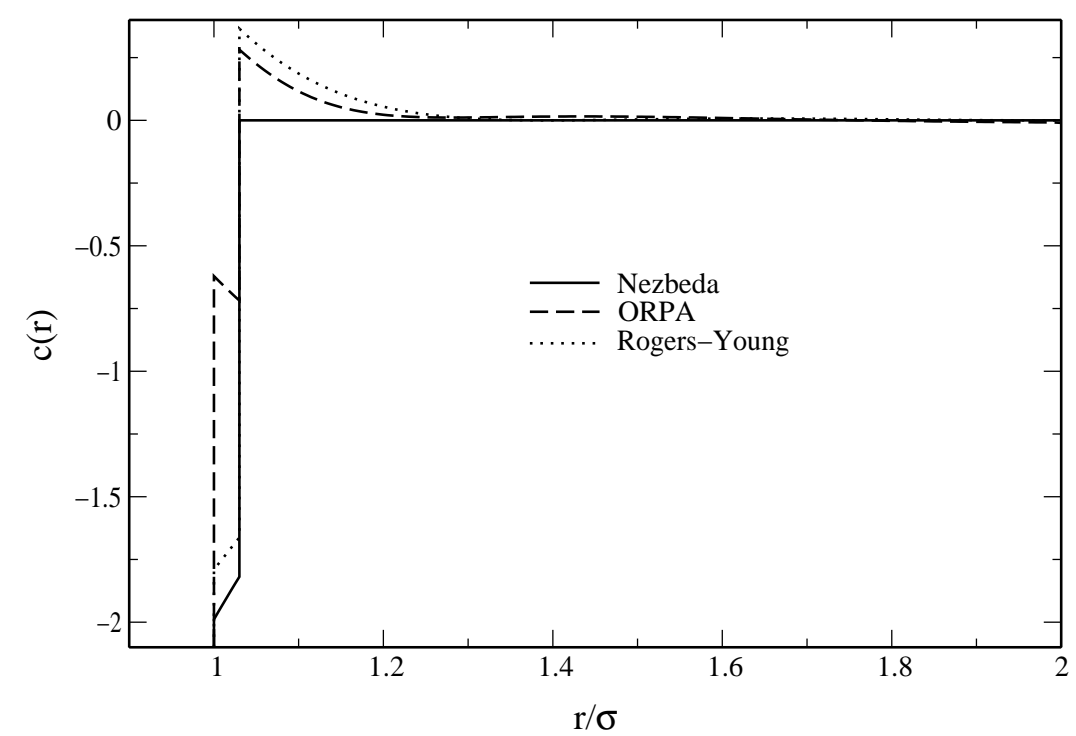

Figure 11. Comparison of the Nezbeda, ORPA and RY direct correlation functions of a $\lambda=1.03$ square-shoulder liquid at packing fraction $\eta=0.35$ and temperature $T^{*}=1.0$. For clarity, only the region outside the hard core is displayed.

excess free energy of the solid turns out to be artificially high. And as the critical temperature is very sensitive to the details of the free energy, this causes a too high critical temperature for the fcc-fcc transition.

This problem was not observed in the case of the fcc-fcc coexistence of the square-well 
system because there the highly positive value of $c(r ; \eta)$ in the region $\sigma<r<\lambda \sigma$ brings about very low values of $\hat{\eta}$, irrespective of the tail of the direct correlation function outside $r=\lambda \sigma$. In the present case, where $c(r ; \eta)$ is negative everywhere but for $r>\lambda \sigma$, taking into account the existence of the tail turns out to be very important. On a more quantitative basis, the non-perturbative approach yields critical temperatures which are even lower than the simulation results [5]. Indeed, in the simulation the critical temperature has the value $T_{c}^{*} \approx 1.5$ and is practically independent of the width of the potential. Our results, however, show a dependence on the width of the repulsive shoulder which is also absent in the previous perturbative approach [13]. This is the same effect as was observed in the non-perturbative approach to the fcc-fcc transition of the square-well potential [25].

\section{Conclusions}

In conclusion we have discussed the thermodynamics, structure and phase transitions in squareshoulder and square-well systems using different variants of approximative theories such as ORPA and Rogers-Young closures and 'exact' computer simulations. Within the modifiedweighted-density approximation we have predicted isostructural solid-solid transitions with the full liquid input, i.e., avoiding a perturbative density functional approach.

We finish with a couple of remarks: first it would be interesting to include polydispersity in the model potential in order to describe real colloidal samples appropriately. In our case, three different kinds of polydispersity are relevant: size polydispersity affecting the core diameter $\sigma$ of the colloidal particles, as well as polydispersity in the range $\lambda$ and in the depth/height $\epsilon$ of the interaction. The Rogers-Young closure [29] and other theories [30,31] can be suitably generalized to treat polydispersity. It is clear that polydispersity will considerably affect the existence and the actual location of the solid-solid critical point of the isostructural transition. Second, even more complicated model potentials exhibiting further barriers following the attractive part [32] promise unusual structural correlations and a rich phase transition scenario with both gas-liquid and solid-solid critical points and more 'exotic' solid phases such as one-component quasicrystals [33]. It would be interesting to apply our approximative scheme to such potentials. Work along these lines is in progress.

\section{Acknowledgments}

This work was supported by the Österreichische Forschungsfond under Project Nos P11194-PHY and P13062-TPH and the Österreichische Nationalbank under Project No 6241. AL acknowledges financial support by a 'Förderungsstipendium der Technischnaturwissenschaftlichen Fakultät' of the TU Wien and by the Deutsche Forschungsgemeinschaft within the SFB 237. AL would like to thank for its hospitality the Forschungszentrum Jülich, where part of this work was performed. GK is indebted to Dr Giorgio Pastore (Trieste) for useful hints, helpful discussions and for providing a copy of his ORPA code.

\section{References}

[1] Vega L, de Miguel E, Rull L F, Jackson G and McLure I A 1992 J. Chem. Phys. 962296

[2] Kahl G and Hafner J 1982 Phys. Chem. Liq. 12109

[3] Hansen J-P and McDonald I R 1986 Theory of Simple Liquids 2nd edn (London: Academic)

[4] Gopala R V and Debnath D 1990 Colloid Polym. Sci. 268604

Bergenholtz J and Wagner N J 1994 Indust. Eng. Chem. Res. 332391

Bergenholtz J and Wagner N J 1994 Langmuir 111559 
[5] Bolhuis P and Frenkel D 1997 J. Phys.: Condens. Matter 9381

[6] Bolhuis P and Frenkel D 1994 Phys. Rev. Lett. 722221

[7] Bergenholtz J, Wu P, Wagner N J and D'Aguanno B 1987 Mol. Phys. 87331

[8] Andersen H C, Chandler D and Weeks J D 1972 J. Chem. Phys. 563812

[9] Andersen H C and Chandler D 1972 J. Chem. Phys. 571918

[10] Andersen H C, Chandler D and Weeks J D 1976 Adv. Chem. Phys. 34105

[11] Rogers F J and Young D A 1984 Phys. Rev. A 30999

[12] Nezbeda I 1977 Czech. J. Phys. B 27247

[13] Denton A R and Löwen H 1997 J. Phys.: Condens. Matter 9 L1

[14] For another application of the ORPA, see Löwen H and Baier T 1990 Phys. Rev. B 414435

[15] Weeks J D, Chandler D and Andersen H C 1971 J. Chem. Phys. 545237

Andersen H C, Weeks J D and Chandler D 1971 Phys. Rev. A 41597

[16] Pastore G, Matthews F, Akinlade O and Badirkhan Z 1995 Mol. Phys. 84653

[17] Pastore G, Akinlade O, Matthews F and Badirkhan Z 1998 Phys. Rev. E 57460

[18] Oberle R and Beck H 1979 Solid State Commun. 32959

[19] Wertheim M S 1963 Phys. Rev. Lett. 10321

Wertheim M S 1964 J. Math. Phys. 5643

Thiele E 1963 J. Chem. Phys. 39474

[20] Verlet L and Weis J-J 1972 Phys. Rev. A 5939

[21] Verlet L and Weis J-J 1974 Mol. Phys. 28665

[22] Smith W R, Henderson D and Tago Y 1977 J. Chem. Phys. 675308

[23] Rosenfeld Y and Ashcroft N W 1979 Phys. Rev. A 201208

[24] Watzlawek M, Löwen H and Likos C N 1998 J. Phys.: Condens. Matter 108189

[25] Likos C N and Senatore G 1995 J. Phys.: Condens. Matter 76797

[26] Allen M P and Tildesley D J 1987 Computer Simulations of Liquids (Oxford: Clarendon)

[27] Parola A and Reatto L 1995 Adv. Phys. 44211

[28] Denton A R and Ashcroft N W 1989 Phys. Rev. A 394701

[29] D'Aguanno B and Klein R 1992 Phys. Rev. A 467652

[30] Lado F 1999 New Approaches to Problems in Liquid State Theory (NATO Science Series C: Mathematical and Physical Sciences, vol 529) ed C Caccamo, J-P Hansen and G Stell (Dordrecht: Kluwer)

[31] Leroch S, Kahl G and Lado F 1999 Phys. Rev. E 596937

[32] Denton A R and Löwen H 1997 J. Phys.: Condens. Matter 98907

[33] Denton A R and Löwen H 1998 Phys. Rev. Lett. 81469 